Introduction The genital infection by the HPV is among the most frequent sexually transmitted diseases (STD) worldwide, and it may result in lesions that can lead to the carcinogenesis of the genital tract. However, other factors may be associated with the onset or progression of the tissue malignancy process, such as the MCPyV, which may present oncogenic profile in the epithelial tissue. This study aims to investigate the presence of MCPyV and HPV in malignant lesions of the male and female genital tract, in order to contribute to the elucidation of the role of these viruses in the cellular malignancy process and to the epidemiological knowledge regarding the prevalence of both viruses in neoplastic lesions.

Methods This is a cross-sectional study evaluating the prevalence of HPV and MCPyV infection in samples of cervical carcinoma and penile cancer. To date, we have obtained 112 samples of penile carcinoma and 31 samples of cervical carcinoma. So, we aim to detect the presence of HPV DNA by the polymerase chain reaction (PCR) technique using the generic primers MY09/MY11; to genotype HPVs by specific PCR to the E6 gene; to detect and quantify DNA of the MCPyV by the Nested PCR technique and real-time PCR; to investigate the presence of truncation mutations in the major $\mathrm{T}$ antigen of MCPyV.

Results Results are partial. To date, all the male samples were analysed. We verified the presence of HPV in $54(48.2 \%)$ of these samples, in which the most prevalent type was the HPV16 (66\%). The cervical carcinoma samples are still under analysis.

Conclusion The collection of cervical neoplasia samples is still being performed. In 2015, our research group found a case of multiple infection by HPV, MCPyV and Epstein-Barr virus in a case of squamous cell carcinoma of the penis in Rio de Janeiro. This was the first report of the presence of MCPyV in this type of penile lesion. Thus, we look forward to find results that contribute to the presence of $\mathrm{MCPyV}$ in genital malignant lesions and to elucidate its role in the oncogenic pathway of malignant lesions.

\section{P3.237 DIRECT DETECTION OF MOSAIC PENA IN CLINICAL SAMPLES CONTAINING NEISSERIA GONORRHOEAE}

${ }^{1}$ PFG Wolffs, 'BMJW van der Veer, 1,2CJPA Hoebe, ${ }^{1,2}$ Dukers-Muijrers NHTM, '1van Alphen LB, ${ }^{1} \mathrm{IHM}$ van Loo. ${ }^{1}$ Maastricht University Medical Centre, Care and Public Health Research Institute (CAPHRI), Maastricht, the Netherlands; ${ }^{2}$ Public Health Service South Limburg, Department of Sexual Health, Infectious Diseases and Environmental Health, Geleen, the Netherlands

\subsection{6/sextrans-2017-053264.472}

Introduction Current surveillance of antibiotic resistance in Neisseria gonorrhoeae (NG) relies heavily on the culture of NG. However, culture of NG is challenging due to demanding nutritional and growth requirements of this micro-organism. As a result, surveillance data are limited to only cultured strains while of $>50 \%$ of Dutch NG positive patients no NG is cultured (data from Dutch Gonococcal Surveillance Program). In this study we compared results from direct detection of mosaic penA with detection of cultured strains to investigate feasibility of direct molecular resistance surveillance.

Methods A convenience sample of 106 NG positive samples of which positive NG culture results were available (46 urine, 9 genital swabs, 35 anorectal swabs and 16 oropharyngeal swabs) were collected between 2013-2015. Presence of mosaic penA was determined by real-time PCR. All positive findings were confirmed with sequencing. MICs on cultured NG were determined using E-tests.

Results LOD determinations of the in-house mosaic penA PCR in comparison to routine NAAT (using COBAS 4800, Roche Diagnostics) showed that the mosaic penA assay was slightly less sensitive than the commercial NAAT. In samples with very low NG loads, mosaic penA detection might be false-negative. Of $106 \mathrm{NG}$ positive samples, 11 samples showed the presence of mosaic penA (6 urine, 4 oropharyngeal and 1 anorectal swab). Of these 11 samples, NG isolates were re-cultured from 8 samples and all isolates contained the mosaic penA gene. MIC values for ceftriaxone varied between 0.016 and $0.094 \mathrm{mg} / \mathrm{L}$ and thus no reduced susceptibility was observed. Although cross-detection with mosaic penA from $N$. meningitidis is possible, no evidence of this was shown in this study.

Conclusion In conclusion, this study indicates that detection of mosaic penA directly from clinical samples is feasible and that results match detection of penA from clinical isolates obtained from these samples. Direct detection of antibiotic resistance genes would show an insight in resistance surveillance of strains that are not or cannot be cultured.

\section{P3.238 SYPHILIS INFECTION STATUS AND THE ASSOCIATED FACTORS AMONG PARTNERS OF SYPHILIS-INFECTED PREGNANT WOMEN IN SHENZHEN, CHINA}

$X$ Wu, F Yang, L Lan, Y Guan, C Zhang, T Feng. Shenzhen Centre for Chronic Disease Control, Shenzhen, China

\subsection{6/sextrans-2017-053264.473}

Introduction The goal of this study is to investigate syphilis infection status among partners of syphilis-infected pregnant women, and to analyse the associated factors from the perspective of both pregnant women and their partners.

Methods This study was based on Shenzhen Program for Prevention of Mother-to-Child Transmission of Syphilis. Followup survey and contact tracing were conducted among syphilisinfected pregnant women diagnosed from 2008 to 2015. Both toludine red unheated serum test (TRUST) and treponema pallidum particle agglutination (TPPA) were applied to partners attending antenatal care clinics. Structural questionnaire was used to collect information. Binary logistic regression model was applied to analyse the factors associated with partners' syphilis infection.

Results During the studied years, 6318 pregnant women were identified with syphilis infection and 5351 partners of these women visited the antenatal care clinics and took the serological testing. From the laboratory record and clinical examination results, 1148 partners were diagnosed with syphilis infection. The infection rate decreased from $26.23 \%$ in 2008 to $15.07 \%$ in 2015 . The partners were more likely to have syphilis infection when pregnant women with TRUST titers $\geq 1$ 8 or at the early syphilis stage. The partners had more risk of syphilis infection when they had multiple sex partners.

Conclusion Syphilis infection rate among partners of syphilisinfected pregnant women is high. Pregnant women's levels of TRUST titer and disease stage as well as partners' sexual behaviours were important factors associated with partners' syphilis infection. Partner notification and contact tracing is an important way to identify syphilis patients. 